\title{
Persistent depressive symptoms and cognitive decline in older adults
}

\author{
Fanfan Zheng, Baoliang Zhong, Xiaoyu Song and Wuxiang Xie
}

\section{Background}

Little is known about the effect of persistent depressive symptoms on the trajectory of cognitive decline.

\section{Aims}

We aimed to investigate the longitudinal association between the duration of depressive symptoms and subsequent cognitive decline over a 10-year follow-up period.

\section{Method}

The English Longitudinal Study of Ageing cohort is a prospective and nationally representative cohort of men and women living in England aged $\geq 50$ years. We examined 7610 participants with two assessments of depressive symptoms at wave 1 (2002-2003) and wave 2 (2004-2005), cognitive data at wave 2 and at least one reassessment of cognitive function (wave 3 to wave 7, 20062007 to 2014-2015).

\section{Results}

The mean age of the 7610 participants was $65.2 \pm 10.1$ years, and $57.0 \%$ were women. Of these, 1157 (15.2\%) participants had episodic depressive symptoms and 525 participants (6.9\%) had persistent depressive symptoms. Compared with participants without depressive symptoms at wave 1 and wave 2, the multivariable-adjusted rates of global cognitive decline associated with episodic depressive symptoms and persistent depressive symptoms were faster by -0.065 points/year $(95 \% \mathrm{Cl}$ -0.129 to -0.000 ) and -0.141 points/year $(95 \% \mathrm{Cl}-0.236$ to $-0.046)$, respectively $(P$ for trend $<0.001)$. Similarly, memory, executive and orientation function also declined faster with increasing duration of depressive symptoms (all $P$ for trend $<0.05$ )

\section{Conclusions}

Our results demonstrated that depressive symptoms were significantly associated with subsequent cognitive decline over a 10-year follow-up period. Cumulative exposure of long-term depressive symptoms in elderly individuals could predict accelerated subsequent cognitive decline in a dose-response pattern.

\section{Declaration of interest}

None.

\section{Keywords}

Depressive symptoms; cognitive decline; trajectory; ELSA.

\section{Copyright and usage}

(c) The Royal College of Psychiatrists 2018
Depressive symptoms and cognitive impairment are two of the most prevalent psychiatric conditions strongly associated with poor quality of later life and a high social burden. ${ }^{1-4}$ Because of its significant clinical and public health importance, the relationship between late-life depressive symptoms and subsequent cognitive decline indicative of dementia has become an increasing focus of contemporary research. A series of cohort studies have suggested that depressive symptoms are associated with cognitive decline or dementia, ${ }^{5-13}$ whereas several other studies reported inconsistent findings. ${ }^{14-17}$ Studies reporting these inconsistent findings have largely focused on the association between a single-time (mostly baseline) assessment of depressive symptoms and cognitive decline. ${ }^{5-8,12-16}$ However, as depressive symptoms tend to fluctuate over time, ${ }^{18,19}$ it is likely that these studies may not have fully captured the persistent effect of these symptoms on cognitive decline, affecting our understanding of this relationship. Only a small number of longitudinal studies using repeated assessments of depressive symptoms have been conducted to date, and these studies have typically used small sample sizes over a short time period, ${ }^{10,11}$ or in a particular subpopulation, such as women, ${ }^{9}$ which may limit the generalisability of the results. Moreover, most prior studies have explored the association between baseline depressive symptoms and incident cognitive impairment, ${ }^{5-7,10-13,16}$ rather than the association between depressive symptoms and the trajectory of cognitive decline. The trajectory of cognitive decline, which consists of several assessments of cognitive function, is of great importance for evaluating and monitoring the progression of cognitive deterioration and could therefore be more informative for early intervention.

The English Longitudinal Study of Ageing (ELSA) has involved multiple phases of data collection, representing a golden opportunity for exploring the association between late-life depressive symptoms and the trajectory of subsequent cognitive decline. The objectives of the present study were (a) to determine whether depressive symptoms predict cognitive decline in an elderly population at baseline, and (b) to examine whether people reporting episodic or persistent depressive symptoms show a similar or different association with subsequent cognitive decline.

\section{Method}

\section{Study population}

This study used data from wave 1 (2002-2003) to wave 7 (20142015) of the ELSA study, which is a prospective and nationally representative cohort of men and women living in England aged 50 years and over. ${ }^{20} \mathrm{~A}$ detailed description of the goals, design and methods of the ELSA has been published elsewhere. ${ }^{21}$ A flowchart of the cohort is shown in Supplementary Figure 1 available at https://doi.org/10.1192/bjp.2018.155. A total of 12099 participants took part in the wave 1 survey of the ELSA. Of these, 3226 participants were excluded from this study because they did not attend the wave 2 survey $(n=2706)$, did not complete the cognitive tests at wave 1 or wave $2(n=502)$ or had self-reported doctor-diagnosed dementia at wave 1 or wave $2(n=18)$. An additional 1263 individuals were excluded because of loss to follow-up from wave 3 to wave 7 . The remaining 7610 participants ( 3272 men and 4338 women) with complete baseline data (wave 2) and at least one reassessment of cognitive function (wave 3 to wave 7 ) were included in the analyses reported here.

The ELSA study was approved by the London Multicentre Research Ethics Committee (MREC/01/2/91) and informed consent was obtained from all participants. 


\section{Cognitive assessments}

The cognitive assessments were conducted as described previously. ${ }^{22,23}$ The memory function of each participant was measured with immediate and delayed recall of ten unrelated words. Both immediate and delayed recall scores ranged from 0 to 10 , with higher scores indicating better memory performance. Immediate and delayed recall tests have been shown to have good construct validity and consistency. ${ }^{24}$ A composite memory score was created by summing the scores of the individual memory tests. Executive function was assessed with a verbal fluency task, in which individuals were required to verbally name as many animals as they could in $60 \mathrm{~s}$. Because of the well-documented reliability and validity of this task, it has already been utilised as a solid indicator of executive function within the ELSA population. ${ }^{25}$ The total score on the animal-naming test was the total number of words produced, excluding repetitive words and words outside the animal category. Orientation to time was assessed by asking four questions (one point each for day of month, month, year and day of week). A global cognitive score was created by summing the individual scores on the memory, executive function and orientation assessments. Generally, higher scores indicate better cognitive function.

\section{Depressive symptoms}

Depressive symptoms were measured with the eight-item version of the Center for Epidemiologic Studies Depression Scale (CES-D), a widely used self-report measure of depressive symptoms, used to identify people at risk of depression in population-based studies. ${ }^{22}$ Participants were asked to think about the past week and the feelings they experienced and to indicate whether each of the following statements was true for them much of the time during the past week: you felt depressed; you felt that everything was an effort; your sleep was restless; you were happy; you felt lonely; you enjoyed life; you felt sad; you could not get going. There were two response options: yes or no. This version of the CES-D has an internal consistency and factor structure that are comparable with longer versions of the scale. ${ }^{26}$ As in previous studies, we used a score of $\geq 4$ to define participants with elevated depressive symptoms. ${ }^{27}$ To derive a duration of depressive symptoms score, we totalled the number of occasions an individual was recorded as being a case at each wave, resulting in a score range from 0 (never a case) to 2 (a case at both wave 1 and wave 2). The sum of CES-D scores at wave 1 and wave 2, ranging from 0 to 16 , was used when modelled as a continuous measure. Participants reporting depressive symptoms at wave 1 or wave 2 were classified as having episodic depressive symptoms, and those reporting depressive symptoms at both waves were classified as having persistent depressive symptoms.

\section{Covariates}

According to previous studies, covariates known to be associated with both depression and cognitive function were selected in our analyses. ${ }^{9,16,28}$ Blood pressure was measured by the nurse in the right arm of each participant while they were in a sitting position, using the Omron HEM-907. ${ }^{29}$ Five minutes elapsed before the first reading was taken. The mean value of three consecutive blood pressure readings was used in our analyses. Hypertension was determined as systolic blood pressure of $\geq 140 \mathrm{~mm} \mathrm{Hg}$ and/or diastolic blood pressure of $\geq 90 \mathrm{~mm} \mathrm{Hg}$, or if the participant was currently using anti-hypertensive drugs. Education level was classified as no qualification, level 1 National Vocational Qualification (NVQ1) or certificate of secondary education, NVQ2 or O-level, NVQ3 or A-level, higher qualification but below degree, and degree level or higher or NVQ4/5. Marital status was classified as single (never married), married, remarried, legally separated, divorced or widowed. We defined cohabitation status as currently living alone or not. Participants were split into two groups: non-smokers (never smoked or ex-smokers) and smokers (current smokers). Alcohol intake was calculated from participant-reported drinking frequency over the previous year (weekly drinking versus occasional or never). Standing height was measured with a portable stadiometer, with participants standing in the centre of the base plate looking straight ahead, and weight was measured by portable electronic scales. ${ }^{29}$ Body mass index was calculated with the following formula: weight $(\mathrm{kg}) /$ height $\mathrm{t}^{2}\left(\mathrm{~m}^{2}\right)$. Diabetes was defined as haemoglobin $\mathrm{A}_{1 \mathrm{c}} \geq 6.5 \%$, fasting blood glucose $\geq 7.00 \mathrm{mmol} / \mathrm{L}$ or current use of anti-diabetic therapy. Measures of chronic disease included lifetime self-reported physician diagnoses of coronary heart disease (angina and heart attack), stroke, chronic lung disease and cancer. The use of antidepressants was measured by asking the participants whether antidepressant medication was recommended by their doctor at wave 2 .

\section{Statistical analysis}

The results are presented as percentages for categorical variables or mean \pm s.d. for continuous variables. The cross-sectional associations between baseline depressive symptoms and cognitive scores at baseline were tested by multiple linear regression models and analyses of covariance. Linear mixed models were used to evaluate the longitudinal associations. Linear mixed models were used to evaluate the longitudinal associations between the sum of CES-D scores (wave 1 and wave 2, range $0-16$ ) and cognitive scores over time. We also ran longitudinal analyses with the duration score of depressive symptoms, with 0 (never a case) as the referent. Linear mixed models use all available data over the follow-up period, take into account the fact that repeated measures on the same participant are correlated with each other and can handle missing data. In these models, both the intercept and the slope were fitted as random effects to account for inter-individual differences at baseline and different rates of change of cognitive function over the follow-up period. The first model included the duration of depressive symptoms $(0,1$ or 2$)$, time (years since baseline), time $\times$ depressive symptoms interaction, age (years) and gender (male or female). The time $\times$ depressive symptoms interaction term indicated differential change of cognitive function by the duration of depressive symptoms from baseline to the end of the study (wave 2 to wave 7). The second model additionally adjusted for baseline body mass index $\left(\mathrm{kg} / \mathrm{m}^{2}\right)$, education $(<\mathrm{NVQ3} / \mathrm{GCE}$ A level or $\geq$ NVQ3/GCE A level), marital status (currently living alone or not), current smoking (yes or no), alcoholic drink (less than once per week or once or more per week), antidepressant medication (yes or no), hypertension (yes or no), diabetes (yes or no), depressive symptoms $\times$ diabetes interaction, coronary heart disease (yes or no), stroke (yes or no), chronic lung disease (yes or no) and cancer (yes or no). Because Katon et al found that the co-occurrence of depression and diabetes was associated with a higher rate of subsequent dementia, ${ }^{28}$ we included the depressive symptoms $\times$ diabetes interaction in the second model.

To enhance the utility of the findings for clinicians, mild cognitive impairment (MCI) was also considered in our analyses. We defined MCI as a global cognitive score <20 (1.5 s.d. below its mean, as proposed by Petersen $e t a l^{30}$ ). The status of MCI (yes or no) was allowed to vary over the follow-up period. Poisson regression with PROC GENMOD (DIST $=$ Poisson and LINK = log) was performed to assess the strength of associations (relative risk) between depressive symptoms and MCI, and generalised estimating equations were used to analyse binary repeated measures data.

We used a multiple imputation, chained-equations method to replace missing data for cognitive assessments during follow-up 
(wave 3 to wave 7), and used all available data from 8873 participants in the sensitivity analyses. Variables used to impute the missing values of cognitive scores included participants' baseline information (age, gender, education, marital status, body mass index, current smoking, alcoholic drink, diabetes and stroke) and baseline cognitive scores. For each longitudinal analysis, we created 20 imputed data-sets and combined the results with the MIANALYZE procedure.

Statistical analyses were performed with SAS software, version 9.4 (SAS Institute Inc., North Carolina, USA; see https://www.sas. com/en_gb/software/sas9.html). All analyses were two-sided, with an alpha value of 0.05 considered as the threshold for statistical significance.

\section{Results}

\section{Baseline characteristics and sample size}

The mean age of the 7610 participants was $65.2 \pm 10.1$ years, and $57.0 \%$ of participants were female. Of these, 1157 (15.2\%) participants were classified as experiencing episodic depressive symptoms and 525 participants (6.9\%) were classified as experiencing persistent depressive symptoms. Table 1 shows the baseline characteristics of participants according to the number of waves with depressive symptoms. In general, compared with those without depressive symptoms, participants who reported a longer duration of depressive symptoms had significantly less favourable characteristics. Study participants reporting a longer duration were older; had higher body mass index; were more likely to be female; had higher percentages of living alone, smoking, diabetes, coronary heart disease, stroke; and chronic lung disease; had lower percentages of higher education and drinking and had worse cognitive function.

Cognitive function was assessed at baseline (wave 2) and reassessed biennially at wave 3 to wave 7 . The cohort size was 7610 (wave 2), 7221 (wave 3), 6225 (wave 4), 5763 (wave 5), 5267 (wave 6) and 4573 (wave 7). The mean follow-up duration was $7.9 \pm 2.9$ years and the mean number of cognitive assessments was $4.8 \pm 1.5$.

\section{Depressive symptoms and cognitive scores (cross- sectional analyses)}

Supplementary Figure 2 shows the multivariable-adjusted cognitive scores according to the number of waves with depressive symptoms. All of the cognitive scores decreased linearly with increasing duration of depressive symptoms (all $P$ for trend $<0.001$ ). As seen in Supplementary Table 1, when modelled as a continuous measure, a one-unit change in the sum of CES-D scores was also associated with the four cognitive scores after adjustment for age and gender (all $P<0.001$ ), and these associations were still significant after multivariable adjustment.

\section{Depressive symptoms and cognitive decline (longitudinal analyses)}

Table 2 shows the longitudinal associations between the sum of CES-D and rate of change in cognitive scores. After multivariable adjustment, a one-unit increment in the sum of CES-D scores was associated with faster declines in global cognitive scores $(-0.012$ points/year, $95 \%$ CI -0.019 to $-0.005, P=0.001$ ), memory scores $(-0.004$ points/year, $95 \% \mathrm{CI}-0.007$ to $-0.002, P=0.002)$, executive function scores $(-0.005$ points/year, $95 \% \mathrm{CI}-0.011$ to $-0.001, P=$ $0.015)$ and orientation scores $(-0.001$ points/year, $95 \%$ CI -0.002 to $-0.001, P<0.001)$.

As shown in Fig. 1, cognitive scores of participants with persistent depressive symptoms significantly deteriorated over time compared with participants without depressive symptoms at wave 1 and wave 2. The multivariable-adjusted rates of global cognitive decline associated with episodic depressive symptoms and persistent depressive symptoms were faster by -0.065 points/year

Table 1 Characteristics of the study participants at baseline (wave 2), according to the number of occasions (wave 1 to wave 2) with depressive symptoms

\begin{tabular}{|c|c|c|c|c|}
\hline \multirow[t]{2}{*}{ Characteristic } & \multicolumn{3}{|c|}{ Number of waves with depressive symptoms } & \multirow[t]{2}{*}{$P$ for trend ${ }^{\mathrm{a}}$} \\
\hline & $0(n=5928)$ & 1 (episodic, $n=1157$ ) & 2 (persistent, $n=525$ ) & \\
\hline Age (years) & $65.0 \pm 10.0$ & $65.5 \pm 10.4$ & $66.6 \pm 11.1$ & $<0.001$ \\
\hline Women (\%) & $3189(53.8)$ & $781(67.5)$ & $368(70.1)$ & $<0.001$ \\
\hline Body mass index $\left(\mathrm{kg} / \mathrm{m}^{2}\right)$ & $27.8 \pm 4.2$ & $28.2 \pm 4.9$ & $28.5 \pm 4.7$ & $<0.001$ \\
\hline Systolic blood pressure (mm Hg) & $136.2 \pm 17.5$ & $135.2 \pm 17.2$ & $136.6 \pm 17.1$ & 0.589 \\
\hline Diastolic blood pressure (mm Hg) & $75.7 \pm 10.1$ & $75.1 \pm 10.5$ & $75.4 \pm 10.3$ & 0.084 \\
\hline Education $\geq$ NVQ3/GCE A-level (\%) & $2129(35.9)$ & $295(25.5)$ & $97(18.5)$ & $<0.001$ \\
\hline Living alone (\%) & $1683(28.4)$ & $502(43.4)$ & $308(58.7)$ & $<0.001$ \\
\hline Current smoking (\%) & $794(13.4)$ & $213(18.4)$ & $144(27.4)$ & $<0.001$ \\
\hline Alcoholic drink $\geq$ once per week (\%) & $3509(59.2)$ & $518(44.8)$ & $190(36.2)$ & $<0.001$ \\
\hline Antidepressant medication (\%) & $48(0.8)$ & $39(3.4)$ & $27(5.1)$ & $<0.001$ \\
\hline Hypertension (\%) & $2463(41.6)$ & 485 (41.9) & $229(43.6)$ & 0.391 \\
\hline Diabetes (\%) & 407 (6.9) & $111(9.6)$ & 59 (11.2) & $<0.001$ \\
\hline Coronary heart disease (\%) & $347(5.9)$ & $98(8.5)$ & $76(14.5)$ & $<0.001$ \\
\hline Stroke (\%) & $127(2.1)$ & $33(2.9)$ & $36(6.9)$ & $<0.001$ \\
\hline Chronic lung disease (\%) & $239(4.0)$ & $75(6.5)$ & $63(12.0)$ & $<0.001$ \\
\hline Cancer (\%) & $303(5.1)$ & $65(5.6)$ & $28(5.3)$ & 0.588 \\
\hline Global cognitive scores & $35.0 \pm 8.5$ & $33.0 \pm 8.8$ & $30.0 \pm 8.7$ & $<0.001$ \\
\hline Memory scores & $10.5 \pm 3.4$ & $9.7 \pm 3.6$ & $8.8 \pm 3.7$ & $<0.001$ \\
\hline Executive function scores & $20.7 \pm 6.4$ & $19.5 \pm 6.3$ & $17.5 \pm 6.1$ & $<0.001$ \\
\hline Orientation scores & $3.79 \pm 0.48$ & $3.76 \pm 0.54$ & $3.68 \pm 0.61$ & $<0.001$ \\
\hline CES-D scores at wave 1 & $0.74 \pm 0.93$ & $3.26 \pm 2.18$ & $5.61 \pm 1.39$ & $<0.001$ \\
\hline CES-D scores at wave 2 & $0.78 \pm 0.93$ & $3.42 \pm 2.11$ & $5.75 \pm 1.36$ & $<0.001$ \\
\hline
\end{tabular}


Table 2 Association between sum of CES-D scores (wave 1 and wave 2 , range $0-16$ ) and rate of change in cognitive scores (points/year): Iongitudinal analyses with linear mixed models

\begin{tabular}{|c|c|c|}
\hline Model terms for cognitive scor & $\beta(95 \% \mathrm{Cl})^{\mathrm{a}}$ & $P$ value \\
\hline \multicolumn{3}{|l|}{ Global cognitive scores } \\
\hline Time & $-0.212(-0.241$ to -0.182$)$ & $<0.001$ \\
\hline CES-D scores & $-0.287(-0.339$ to -0.234$)$ & $<0.001$ \\
\hline CES-D scores $\times$ time & $-0.012(-0.019$ to -0.005$)$ & 0.001 \\
\hline \multicolumn{3}{|l|}{ Memory scores } \\
\hline Time & $-0.083(-0.094$ to -0.072$)$ & $<0.001$ \\
\hline CES-D scores & $-0.101(-0.121$ to -0.081$)$ & $<0.001$ \\
\hline CES-D scores $\times$ time & $-0.004(-0.007$ to -0.002$)$ & 0.002 \\
\hline \multicolumn{3}{|l|}{ Executive function scores } \\
\hline Time & $-0.091(-0.114$ to -0.069$)$ & $<0.001$ \\
\hline CES-D scores & $-0.178(-0.219$ to -0.138$)$ & $<0.001$ \\
\hline CES-D scores $\times$ time & $-0.005(-0.011$ to -0.001$)$ & 0.015 \\
\hline \multicolumn{3}{|l|}{ Orientation scores } \\
\hline Time & $-0.011(-0.013$ to -0.008$)$ & $<0.001$ \\
\hline CES-D scores & $-0.006(-0.009$ to -0.003$)$ & $<0.001$ \\
\hline CES-D scores $\times$ time & $-0.001(-0.002$ to -0.001$)$ & $<0.001$ \\
\hline \multicolumn{3}{|c|}{$\begin{array}{l}\text { CES-D, Center for Epidemiologic Studies Depression. } \\
\text { a. Adjusted for baseline age, gender, body mass index, education, marital status, current } \\
\text { smoking, alcoholic drink, antidepressant medication, hypertension, diabetes, depressive } \\
\text { symptoms } \times \text { diabetes interaction, coronary heart disease, stroke, chronic lung disease } \\
\text { and cancer. }\end{array}$} \\
\hline
\end{tabular}

( $95 \% \mathrm{CI}-0.129$ to -0.000 ) and -0.141 points/year (95\% CI -0.236 to -0.046), respectively, compared with participants without depressive symptoms ( $P$ for trend $<0.001$; Table 3 ). Similarly, memory, executive and orientation function also declined faster with increasing duration of depressive symptoms (all $P$ for trend $<0.05$; Table 3). Compared with the reference category, the multivariable-adjusted rate of memory decline associated with persistent depressive symptoms was faster by -0.048 points/year $(95 \%$ CI -0.084 to -0.013 ), and the rates of executive and orientation function decline were faster by -0.074 points/year ( $95 \%$ CI -0.146 to $-0.002)$ and -0.014 points/year $(95 \%$ CI -0.023 to -0.006$)$, respectively (Table 3 ).

\section{Depressive symptoms and $\mathrm{MCl}$ (longitudinal analyses)}

As shown in Supplementary Table 2, compared with participants without depressive symptoms, episodic and persistent depressive symptoms were associated with a 13 and $28 \%$ greater risk of MCI, respectively, after multivariable adjustment.

\section{Non-response analyses}

A total of 1263 participants (14.2\%) with complete baseline data were excluded from the study because they were lost to follow-up. This group of excluded participants were older; had higher levels of systolic blood pressure; higher percentages of living alone, current smoking, diabetes, coronary heart disease, stroke, chronic lung disease, cancer and depressive symptoms; had lower percentages of higher education and drinking and had worse cognitive function (see Supplementary Table 3).
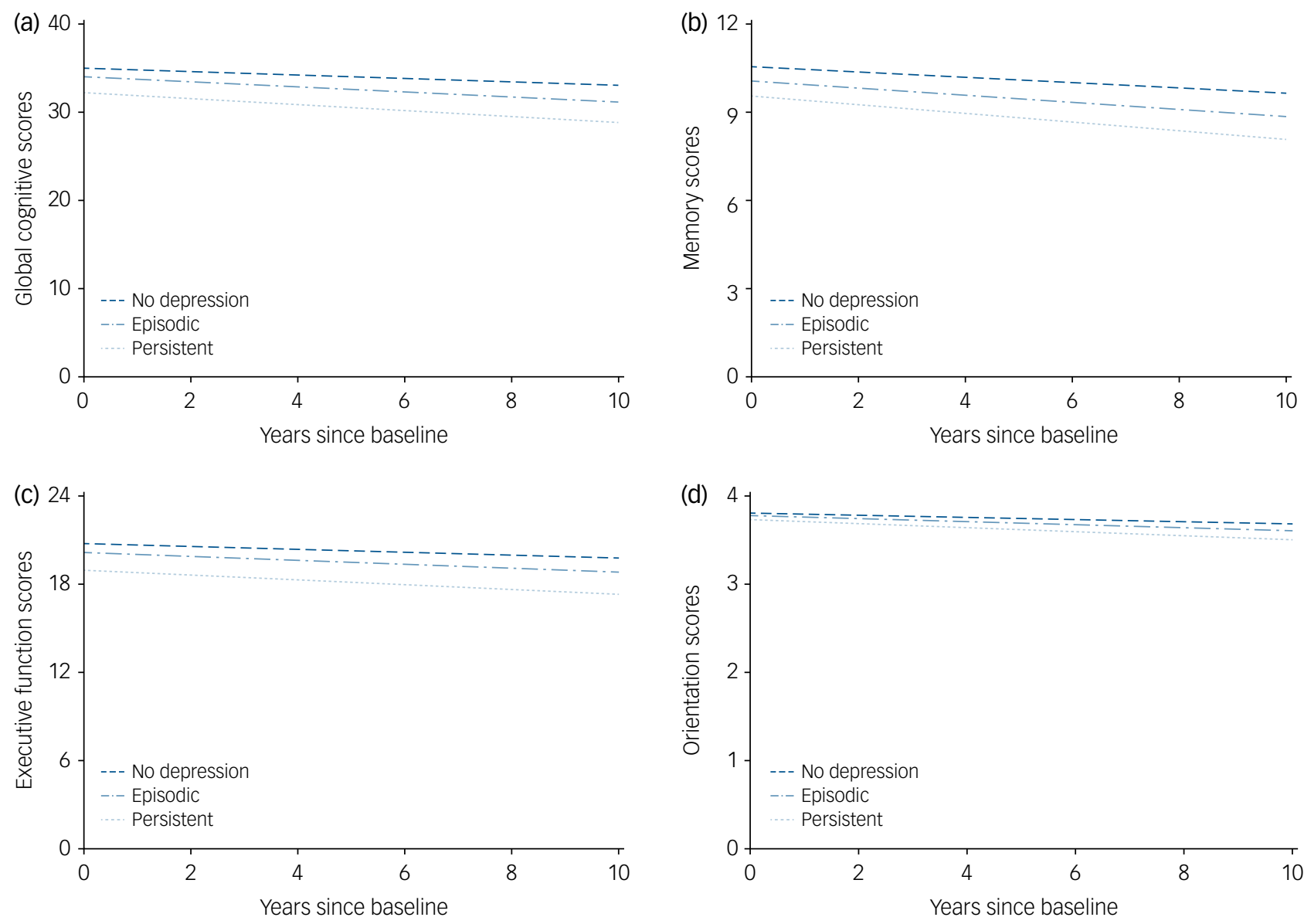

Fig. 1 The trajectories of cognitive scores by the number of waves with depressive symptoms (wave 1 to wave 2), adjusted for baseline age, gender, body mass index, education, marital status, current smoking, alcoholic drink, antidepressant medication, hypertension, diabetes, depressive symptoms $\times$ diabetes interaction, coronary heart disease, stroke, chronic lung disease and cancer. 
Table 3 Mean difference in the rate of change in cognitive scores (points/year) comparing the number of waves with depressive symptoms (wave 1 to wave 2): Iongitudinal analyses with linear mixed models

\begin{tabular}{|c|c|c|c|c|}
\hline & \multicolumn{3}{|c|}{$\begin{array}{l}\text { Mean difference }(95 \% \mathrm{Cl}) \text { in rate of change by number of waves with depressive } \\
\text { symptoms }\end{array}$} & \multirow[t]{2}{*}{$P$ for trend } \\
\hline & $0(n=5928)$ & 1 (episodic, $n=1157$ ) & 2 (persistent, $n=525$ ) & \\
\hline \multicolumn{5}{|c|}{ Global cognitive scores } \\
\hline Model $1^{\mathrm{a}}$ & 0.000 (ref) & $-0.065(-0.130$ to -0.001$)$ & $-0.142(-0.238$ to -0.047$)$ & $<0.001$ \\
\hline Model $2^{b}$ & 0.000 (ref) & $-0.065(-0.129$ to -0.000$)$ & $-0.141(-0.236$ to -0.046$)$ & $<0.001$ \\
\hline \multicolumn{5}{|c|}{ Memory scores } \\
\hline Model $1^{a}$ & 0.000 (ref) & $-0.025(-0.049$ to -0.001$)$ & $-0.049(-0.085$ to -0.013$)$ & 0.001 \\
\hline Model $2^{\mathrm{b}}$ & 0.000 (ref) & $-0.025(-0.049$ to -0.001$)$ & $-0.048(-0.084$ to -0.013$)$ & 0.002 \\
\hline \multicolumn{5}{|c|}{ Executive function scores } \\
\hline Model $1^{\mathrm{a}}$ & 0.000 (ref) & $-0.030(-0.078$ to 0.019$)$ & $-0.075(-0.147$ to -0.003$)$ & 0.025 \\
\hline Model $2^{\mathrm{b}}$ & 0.000 (ref) & $-0.029(-0.078$ to 0.019$)$ & $-0.074(-0.146$ to -0.002$)$ & 0.027 \\
\hline \multicolumn{5}{|c|}{ Orientation scores } \\
\hline Model $1^{a}$ & 0.000 (ref) & $-0.005(-0.011$ to 0.001$)$ & $-0.014(-0.023$ to -0.006$)$ & $<0.001$ \\
\hline Model $2^{\mathrm{b}}$ & 0.000 (ref) & $-0.005(-0.011$ to 0.001$)$ & $-0.014(-0.023$ to -0.006$)$ & $<0.001$ \\
\hline
\end{tabular}

\section{Sensitivity analyses}

Longitudinal results with imputed data $(n=8873)$ were similar to those from the main analyses (see Supplementary Tables 4 and 5). Thus, the effect of missing data was likely to be small.

\section{Discussion}

In this large ongoing cohort study of an elderly British population, depressive symptoms were associated with baseline cognitive function in a cross-sectional analysis. Moreover, depressive symptoms were associated with subsequent cognitive decline over a 10 -year follow-up period in a longitudinal analysis. In addition to this association, we found that the cumulative depressive symptom burden was independently and strongly associated with poorer cognitive performance and could, in a dose-response pattern, predict accelerated subsequent cognitive decline.

This study used previous assessments of depressive symptoms as a baseline to investigate the temporal association between persistent depressive symptoms and the trajectory of subsequent cognitive decline in a large general population, using depressive symptom data collected at wave 1 and wave 2 and cognitive decline data from wave 2 to wave 7 . Several cohort studies have been conducted to explore the association between cumulative depressive symptoms and cognitive decline. ${ }^{9,31,32}$ However, these studies were unable to confirm the temporal relations involved because it could not be determined with certainty whether a change in mood status preceded the development of cognitive decline based on data collected simultaneously during the same follow-up period. Although a small number of studies have examined the fluctuating nature of depressive symptoms over time, using multiple depressive assessments for statistical analysis, they have typically used incident cognitive decline as the main outcome measure and failed to show an effect on subsequent cognitive trajectory. ${ }^{10,11}$ Apart from the methodological differences mentioned above, our results were largely compatible with previous findings, ${ }^{9-11}$ demonstrating not only a significant association of depressive symptoms with cognitive decline, but also a greater effect of persistent depressive symptoms on subsequent cognitive deterioration. Importantly, our results are also supported by a recent study with a clinically depressed sample. ${ }^{33}$ Nevertheless, it should be noted that only a weak association between depressive symptoms and cognitive decline was observed, in accordance with previous studies. ${ }^{8,32}$ Although depressive symptoms may have caused only miniscule annual changes in cognitive function, the phenomenon could play an important role in cognitive decline. As reported in a previous study, even minor differences in cognition can lead to a substantially increased risk of dementia over several years. ${ }^{34}$ Because there is currently no cure for dementia, early detection and intervention based on modifiable predictors, such as depressive symptoms, may offer an effective approach for the prevention of cognitive decline, delaying the progression to dementia and reducing its prevalence and associated public health burden.

Depression and cognitive impairment can have severe consequences, including reduced quality of life, elevated needs of social services and increased mortality, and are among the most important mental health problems among elderly people. ${ }^{1-4}$ Late-onset depression and $\mathrm{MCI}$ occur together at a rate of up to $63 \%$, suggesting a close association between the two disorders. ${ }^{35}$ Increasing research attention has focused on elucidating the relationship between depression and subsequent cognitive decline in recent decades, but this research has yielded mixed findings. One possible explanation for the discrepancy in previous findings might be related to the fluctuating nature of depressive symptoms over time. As mentioned above, most previous studies have been limited by their use of a single assessment of depressive symptoms, potentially resulting in underestimation of the association. As such, our study adds more evidence to previous research by exploring the influence of the cumulative burden of repeated measures of depressive symptoms on subsequent cognitive outcomes. In addition, previous studies assessing the association between depressive symptoms and cognitive decline have been heterogeneous in terms of study population, symptom measurement, cognitive assessment, follow-up periods, outcome ascertainment and the reporting of results, which may have contributed to the discrepancies in findings. Furthermore, the magnitude of the dose-response association between the duration of depressive symptoms and faster subsequent cognitive decline was not attenuated by adjusting for these covariates in our linear mixed models, suggesting that depressive symptoms is strongly linked to cognitive decline independently of other social factors and comorbidities. These findings suggest that multiple assessments of depressive symptoms should be taken into account to detect depression and improve prognosis.

Importantly, although our results reveal a significant association of persistent depressive symptoms at wave 1 and wave 2 , with subsequent cognitive decline from wave 2 to wave 7 , the presence of depressive symptoms at subsequent waves and their potential 
effect on cognitive function should be considered. The observed cognitive decline was related not only to depressive symptoms at wave 1 and wave 2, but also to depressive symptoms in later waves. However, because individuals from the persistent depressive symptoms group would be expected to have a higher risk of depressive symptoms in later waves (see Supplementary Table 6), the persistent depressive symptoms at wave 1 and wave 2 could, to some extent, predict accelerated subsequent cognitive decline.

The mechanisms underlying the association between depressive symptoms and cognitive decline remain under debate, and several different mechanisms have been proposed. Accordingly, depressive symptoms could be a risk factor for subsequent cognitive decline, an early manifestation of cognitive deterioration, a reaction to perceived cognitive decline and functional disability or even a symptom of a related risk factor, such as cerebrovascular disease or neurodegenerative changes. ${ }^{11,13,16}$ As summarised by Byers and Yaffe, predominant pathways linking depressive symptoms to cognitive decline include vascular disease, proinflammatory changes and hippocampal atrophy, and those pathways are likely to be multifactorial and not sequential. ${ }^{36}$ It should be noted that, because it is not possible to conduct a randomised trial to test the relationship between depressive symptoms and subsequent cognitive decline, conclusions must be based on observational studies, from which causality cannot be established. However, with the current temporal design, we were able to conclude with confidence that, although a causal relationship remains to be determined, depressive symptoms exhibited years before could predict subsequent cognitive decline, and hence could serve as an indicator for early intervention to prevent further exacerbation of cognitive decline.

To our knowledge, the present study is the largest general population-based study exploring the relationship between depressive symptoms and cognitive decline with a long-term follow-up of 10 years. Moreover, cognitive function was repeatedly measured over a long period, providing a more robust measurement of cognitive deterioration. Our study design enabled us to better measure the cumulative burden of depressive symptoms and to estimate the long-term trajectories of cognitive decline. Nevertheless, our findings should be interpreted cautiously in the context of several potential limitations. First, because our study lacked the clinical diagnosis of dementia during follow-up, we could not analyse the temporal relationship between depressive symptoms and dementia. Second, although we adjusted for a number of potential confounders, we could not rule out the possibility of residual confounding factors, such as anxiety, chronic pain, sleep-related issues and prescribed agents that may affect anxiety and cognition. Genetic susceptibility factors, such as the APOE genotype, are also potential confounders. Currently, the moderating effect of the APOE genotype on the association between depressive symptoms and cognitive outcome remains equivocal, with some studies suggesting a synergistic interaction between APOE genotype and depressive symptoms in elevating the risk of further cognitive decline, ${ }^{6,11}$ whereas others have not supported the existence of such an effect. ${ }^{31,37,38}$ Unfortunately, genetic data are not available in ELSA, so we cannot adjust for the APOE genotype. Third, although we adjusted for the use of antidepressant, it should be noted that the data we used might not represent actual antidepressant use. Fourth, 1263 participants (14.2\%) with complete baseline data were excluded from the study because they were lost to follow-up, potentially causing selection bias. Fifth, the study population was healthier than the original ELSA population, which may threaten the internal validity of estimates, potentially limiting the generalisability of our findings to the English population. However, sensitivity analyses with multiple imputation methods revealed similar results, indicating that the effect of attrition was minimal. In addition, cognition was assessed by isolated tasks in this study. Broader assessment of neuropsychological cognitive domain performance may have produced different results. Besides, individuals with depressive symptoms were not identified by a clinical diagnosis of depression made by a psychiatrist according to symptom criteria. The CES-D mainly assesses the depressive symptoms in the past week, which might lead to underestimation of the presence of depressive symptoms. Finally, although we identified the detection of depressive symptoms at both wave 1 and wave 2 as persistent depressive symptoms, it is still possible that what we captured are actually two separate episodes of depressive symptoms. In this case, the effect of persistent depressive symptoms on subsequent cognitive trajectory might represent the cumulative burden of recurrent depressive symptoms.

In conclusion, this study demonstrated that depressive symptoms were significantly associated with both baseline cognitive function and subsequent cognitive decline over a 10-year followup period. Cumulative exposure of long-term depressive symptoms in elderly individuals could predict accelerated subsequent cognitive decline in a dose-response pattern. Careful monitoring of depressive symptoms in older adults may benefit early intervention and treatment of those symptoms, delaying the progression of cognitive impairment and the development of late-onset dementia.

Fanfan Zheng Associate Research Fellow, Brainnetome Center Institute of Automation, Chinese Academy of Sciences, China and Visiting Research Fellow, Institute of Cognitive Neuroscience, University College London, UK; Baoliang Zhong, Associate Professor, Department of Geriatric Psychiatry, Affiliated Wuhan Mental Health Center, Tongji Medical College of Huazhong University of Science \& Technology, China; Xiaoyu Song, Assistant Professor, Department of Population Health Science and Policy, Icahn School of Medicine at Mount Sinai, USA; Wuxiang Xie, Associate Research Fellow, Peking University Clinical Research Institute, Peking University Health Science Center, China and Newton International Fellow, Department of Epidemiology and Biostatistics, School of Public Health, Imperial College London, UK

Correspondence: Wuxiang Xie, PhD, Peking University Clinical Research Institute, Peking University Health Science Center, No. 38 Xueyuan Road, Haidian District, Beijing, 100191, China. Email: xiewuxiang@hsc.pku.edu.cn

First received 22 Nov 2017, final revision 25 Apr 2018, accepted 11 Jul 2018

\section{Supplementary material}

Supplementary material is available online at https://doi.org/10.1192/bjp.2018.155.

\section{Funding}

This study was funded by the National Natural Science Foundation of China (project no. 81601176), the Beijing Natural Science Foundation (project no. 7182108) and the Newton International Fellowship from the Academy of Medical Sciences (project no. NIF001-1005P56804). The funding source had no role in the design and conduct of the study; collection, management, analysis and interpretation of the data; preparation, review or approval of the manuscript and decision to submit the manuscript for publication.

\section{Acknowledgments}

We thank the original data creators, depositors, copyright holders, the funders of the Data collections and the UK Data Archive for the use of data from English Longitudinal Study of Ageing: waves 0-7, 1998-2015. The original data creators, depositors or copyright holders bear no responsibility for the current analysis or interpretation.

\section{References}

1 Byers AL, Yaffe K, Covinsky KE, Friedman MB, Bruce ML. High occurrence of mood and anxiety disorders among older adults: the National Comorbidity Survey Replication. Arch Gen Psychiatry 2010; 67(5): 489-96.

2 Yaffe K, Middleton LE, Lui LY, Spira AP, Stone K, Racine C, et al. Mild cognitive impairment, dementia, and their subtypes in oldest old women. Arch Neurol 2011; 68(5): 631-6. 
3 McKenna MT, Michaud CM, Murray CJ, Marks JS. Assessing the burden of disease in the United States using disability-adjusted life years. Am J Prev Med 2005; 28(5): 415-23.

4 Macdonald AJ. ABC of mental health. Mental health in old age. BMJ 1997; 315 (7105): 413-7.

5 Geda YE, Roberts RO, Mielke MM, Knopman DS, Christianson TJ, Pankratz VS, et al. Baseline neuropsychiatric symptoms and the risk of incident mild cognitive impairment: a population-based study. Am J Psychiatry 2014; 171(5): 572-81.

6 Pink A, Stokin GB, Bartley MM, Roberts RO, Sochor O, Machulda MM, et al. Neuropsychiatric symptoms, APOE epsilon4, and the risk of incident dementia: a population-based study. Neurology 2015; 84(9): 935-43.

7 Bassuk SS, Berkman LF, Wypij D. Depressive symptomatology and incident cognitive decline in an elderly community sample. Arch Gen Psychiatry 1998; 55(12): 1073-81.

8 Wilson RS, Barnes LL, Mendes de Leon CF, Aggarwal NT, Schneider JS, Bach J, et al. Depressive symptoms, cognitive decline, and risk of AD in older persons. Neurology 2002; 59(3): 364-70.

9 Zeki Al Hazzouri A, Vittinghoff E, Byers A, Covinsky K, Blazer D, Diem S, et al. Long-term cumulative depressive symptom burden and risk of cognitive decline and dementia among very old women. J Gerontol A Biol Sci Med SCi 2014; 69(5): 595-601.

10 Paterniti S, Verdier-Taillefer MH, Dufouil C, Alperovitch A. Depressive symptoms and cognitive decline in elderly people. Longitudinal study. Br J Psychiatry 2002; 181: 406-10.

11 Geda YE, Knopman DS, Mrazek DA, Jicha GA, Smith GE, Negash S, et al. Depression, apolipoprotein E genotype, and the incidence of mild cognitive impairment: a prospective cohort study. Arch Neurol 2006; 63(3): 435-40.

12 Saczynski JS, Beiser A, Seshadri S, Auerbach S, Wolf PA, Au R. Depressive symptoms and risk of dementia: the Framingham Heart Study. Neurology 2010; 75(1): 35-41.

13 Li G, Wang LY, Shofer JB, Thompson ML, Peskind ER, McCormick W, et al. Temporal relationship between depression and dementia: findings from a large community-based 15-year follow-up study. Arch Gen Psychiatry 2011; 68(9): 970-7.

14 Vinkers DJ, Gussekloo J, Stek ML, Westendorp RG, van der Mast RC. Tempora relation between depression and cognitive impairment in old age: prospective population based study. BMJ 2004; 329(7471): 881.

15 Ganguli M, Du Y, Dodge HH, Ratcliff GG, Chang CC. Depressive symptoms and cognitive decline in late life: a prospective epidemiological study. Arch Gen Psychiatry 2006; 63(2): 153-60.

16 Richard E, Reitz C, Honig LH, Schupf N, Tang MX, Manly JJ, et al. Late-life depression, mild cognitive impairment, and dementia. JAMA Neurol 2013; 70(3): 374-82

17 Comijs HC, van Tilburg T, Geerlings SW, Jonker C, Deeg DJ, van Tilburg W, et al. Do severity and duration of depressive symptoms predict cognitive decline in older persons? Results of the Longitudinal Aging Study Amsterdam. Aging Clin Exp Res 2004; 16(3): 226-32.

18 Judd LL, Akiskal HS, Maser JD, Zeller PJ, Endicott J, Coryell W, et al. A prospective 12-year study of subsyndromal and syndromal depressive symptoms in unipolar major depressive disorders. Arch Gen Psychiatry 1998; 55(8): 694700.

19 Judd LL, Akiskal HS. Delineating the longitudinal structure of depressive illness: beyond clinical subtypes and duration thresholds. Pharmacopsychiatry 2000; 33(1): 3-7.

20 Marmot M, Oldfield Z, Clemens S, Blake M, Phelps A, Nazroo J, et al. English Longitudinal Study of Ageing: Waves 0-7, 1998-2015. [data collection] (27th edn). UK Data Service. SN: 5050, 2017 (http://doi.org/10.5255/UKDA-SN-5050-14).
21 Steptoe A, Breeze E, Banks J, Nazroo J. Cohort profile: the English longitudinal study of ageing. Int J Epidemiol 2013; 42(6): 1640-8.

22 Zheng F, Xie W. High-sensitivity C-reactive protein and cognitive decline: the English Longitudinal Study of Ageing. Psychol Med 2018; 48(8): 1381-9.

23 Zheng F, Yan L, Yang Z, Zhong B, Xie W. HbA1C, diabetes and cognitive decline: the English Longitudinal Study of Ageing. Diabetologia 2018; 61(4): 839-48.

24 Baars MA, van Boxtel MP, Dijkstra JB, Visser PJ, van den Akker M, Verhey FR, et al. Predictive value of mild cognitive impairment for dementia. The influence of case definition and age. Dement Geriatr Cogn Disord 2009; 27(2): 173-81.

25 Dregan A, Stewart R, Gulliford MC. Cardiovascular risk factors and cognitive decline in adults aged 50 and over: a population-based cohort study. Age Ageing 2013; 42(3): 338-45.

26 Turvey $C L$, Wallace RB, Herzog R. A revised CES-D measure of depressive symptoms and a DSM-based measure of major depressive episodes in the elderly. Int Psychogeriatr 1999; 11(2): 139-48.

27 Hamer M, Batty GD, Kivimaki M. Risk of future depression in people who are obese but metabolically healthy: the English longitudinal study of ageing. $\mathrm{Mol}$ Psychiatry 2012; 17(9): 940-5.

28 Katon W, Pedersen HS, Ribe AR, Fenger-Gron M, Davydow D, Waldorff FB, et al. Effect of depression and diabetes mellitus on the risk for dementia: a national population-based cohort study. JAMA Psychiatry 2015; 72(6): 612-9.

29 Graig R, Deverill C, Pickering K. Quality control of blood saliva and urine anaIytes. In: Health Survey for England 2004, Methodology and Documentation, vol. 2 (eds Spronston K, Mindell J): 34-41. The Information Centre, 2006.

30 Petersen RC, Smith GE, Waring SC, Ivnik RJ, Tangalos EG, Kokmen E. Mild cognitive impairment: clinical characterization and outcome. Arch Neurol 1999; 56(3): 303-8.

31 Kohler S, van Boxtel MP, van Os J, Thomas AJ, O'Brien JT, Jolles J, et al. Depressive symptoms and cognitive decline in community-dwelling older adults. J Am Geriatr Soc 2010; 58(5): 873-9.

32 van den Kommer TN, Comijs HC, Aartsen MJ, Huisman M, Deeg DJ, Beekman AT. Depression and cognition: how do they interrelate in old age? Am J Geriatr Psychiatry 2013; 21(4): 398-410.

33 Riddle M, Potter GG, McQuoid DR, Steffens DC, Beyer JL, Taylor WD. Longitudinal cognitive outcomes of clinical phenotypes of late-life depression. Am J Geriatr Psychiatry 2017; 25(10): 1123-34.

34 Bozoki A, Giordani B, Heidebrink JL, Berent S, Foster NL. Mild cognitive impairments predict dementia in nondemented elderly patients with memory loss. Arch Neurol 2001; 58(3): 411-6.

35 Panza F, Frisardi V, Capurso C, D'Introno A, Colacicco AM, Imbimbo BP, et al. Late-life depression, mild cognitive impairment, and dementia: possible continuum? Am J Geriatr Psychiatry 2010; 18(2): 98-116.

36 Byers AL, Yaffe K. Depression and risk of developing dementia. Nat Rev Neurol 2011; 7(6): 323-31.

37 Jackson EJ, Rajah S, Morris C, Ballard C, Thomas AJ, O'Brien JT. Is apolipoprotein e4 associated with cognitive decline in depression? Int J Geriatr Psychiatry 2001; 16(4): 436-7.

38 Butters MA, Sweet RA, Mulsant BH, llyas Kamboh M, Pollock BG, Begley AE, et al. APOE is associated with age-of-onset, but not cognitive functioning, in late-life depression. Int J Geriatr Psychiatry 2003; 18(12): 1075-81. 